\title{
Erratum: Communications in Computer and Information Science: A New Scalable Anonymous Authentication Protocol for RFID
}

\author{
Mohammad Shirafkan, Naser Modiri, Mohammad Mansour Riahi Kashani, \\ and Koosha Sadeghi Oskooyee \\ Islamic Azad University, North Tehran branch, \\ Department of Computer Engineering, Tehran, Iran \\ \{Mohammadshirafkan, Nasermodiri\}@yahoo.com, \\ \{M_Riahi_Kashani,K_Sadeghi_oskooyee\}@iau-tnb.ac.ir
}

\begin{abstract}
A. Abd Manaf et al. (Eds.): ICIEIS 2011, Part III, CCIS 253, pp. 639-653, 2011.
(C) Springer-Verlag Berlin Heidelberg 2011
\end{abstract}

\section{DOI 10.1007/978-3-642-25462-8_63}

The paper "Communications in Computer and Information Science: A New Scalable Anonymous Authentication Protocol for RFID” authored by Mohammad Shirafkan, Naser Modiri, Mohammad Mansour Riahi Kashani, and Koosha Sadeghi Oskooyee, DOI 10.1007/978-3-642-25462-8_57, appearing on pages 639-653 of this publication has been retracted due to a severe case of plagiarism. It is a plagiarized version of the paper 'A Privacy-Friendly RFID Protocol Using Reusable Anonymous Tickets', authored by Mahdi Asadpour and Mohammad Torabi Dashti, published in the proceedings of the 10th IEEE International Conference on Trust, Security and Privacy in Computing and Communications (TrustCom 2011), DOI 10.1109/TrustCom.2011.29 and of the master thesis 'An Anonymous RFID Authentication Protocol and its Automated Analysis with OFMC' completed by Mahdi Asadpour at ETH Zurich.

The original online version for this chapter can be found at http://dx.doi.org/10.1007/978-3-642-25462-8_57 